\title{
Comparison of the Blink Reflex and R2 Recovery Cycle Parameters Between Blepharospasm and Healthy Subjects
}

\author{
Maryam Pouranian1 (D), Mohammad Rohani' $^{1}$ (D), Maziar Emamikhah' ${ }^{\text {(D), Gholamali Shahidi }}{ }^{1}$ \\ Mona Ramezani Ghamsari $^{1}$ (D), Mahla Babaie ${ }^{2}$ (D), Mansoureh Babadi ${ }^{3}$ (iD), \\ Mahdi Safdarian 4 (iD), Seyed Amir Hassan Habibi *1 (iD)
}

1. Dept. of Neurology, Rasoul Akram Hospital, Iran University of Medical Sciences, Tehran, Iran

2. Student Research Committee, Iran University of Medical Sciences, Tehran, Iran

3. Student Research Committee, Ahvaz, Jundishapur University of Medical Sciences, Ahvaz, Iran

4. Student Research Committee, Tehran University of Medical Sciences, Tehran, Iran

\section{Article Info \\ 10.30699/jambs.28.131.296 \\ Received: 2019/10/27; \\ Accepted: 2020/09/16; \\ Published Online: 21 Oct 2020 \\ Use your device to scan and read the article online

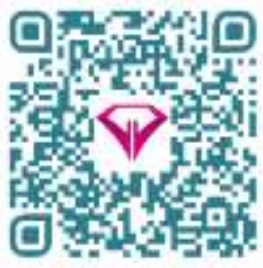

Corresponding Information: Seyed Amir Hassan Habibi, Dept. of Neurology, Rasoul Akram Hospital, Iran University of Medical Science, Tehran, Iran

E-Mail:

habibisaiyedamir@yahoo.com

\section{ABSTRACT}

Background \& Objective: The blink reflex recovery cycle indicates the excitability of interneurons in the brainstem. In this study, we aimed to investigate the blink reflex and R2 recovery cycle in patients with benign essential blepharospasm (BEB).

Materials \& Methods: This prospective case-control study compared the blink reflex and R2 recovery in 18 BEB patients with 18 age- and gender-matched healthy individuals. The blink reflex was measured in a stimulation duration of $0.2 \mathrm{~ms}$, and R1, R2, and R2' were recorded for all subjects. The R2 recovery cycle was measured by stimulation of the supraorbital nerve at four interstimulus intervals (ISI) of 200, 300,500 , and $1000 \mathrm{~ms}$, with the same intensity as the previous test. The R2 recovery index was calculated and compared with the control group via independent sample ttest. Two-tailed P-value less than 0.05 was considered statistically significant.

Results: The blink reflex (R1, R2, and R2' responses) distal latencies and amplitudes in the BEB group were not significantly different from the control group $(P>0.05)$. The $\mathrm{R} 2$ recovery index in all intervals was significantly higher in the BEB group than the control group $(P=0.00)$. The most significant difference between the BEB and control groups regarding the $\mathrm{R} 2$ recovery indices was observed at ISIs of 300 and 200 ms ( 80.36 vs. 16.99 and 75.70 vs. 12.57 , respectively). There was a negative correlation between the patient's age at the onset of disease and the R2 recovery index; however, it was not statistically significant $(P>0.05)$.

Conclusion: Our findings showed that the $\mathrm{R} 2$ recovery reflex was higher in BEB patients than the normal population. Therefore, it can be a helpful index for differentiating BEB cases from psychogenic or malingering ones.

Keywords: Blepharospasm, Blink reflex, Recovery cycle

\section{Introduction}

Benign essential blepharospasm (BEB) is a focal dystonia, characterized by an increased blink rate and sustained involuntary eyelid closure that can be often long-lasting due to the bilateral hyperactivity of the orbicularis oculi muscles (1). The periocular injection of botulinum neurotoxin type A (BTX) has been approved as the first choice of treatment $(2,3)$. The diagnosis of $\mathrm{BEB}$ is based on clinical findings. However, it is sometimes difficult to differentiate it from its mimics, such as eye closure tics and psychogenic blepharospasm (4). There is a primary suspect of secondary blepharospasm in patients with atypical features, such as early and acute onset, constant spasms, or unusual response to BTX injections (5). Therefore, using a test for differentiating idiopathic blepharospasm from other causes of eyelid closure (e.g., psychogenic or malingering) can be helpful.

Some studies have shown an increase in the blink reflex recovery cycle due to enhanced excitability of brainstem interneurons in BEB, which is not influenced by BTX injections (6-8). The blink reflex, elicited by stimulation of the supraorbital nerve, is an electrophysiological analogue of the corneal reflex, which indicates the excitability of brainstem interneurons and comprises two components: R1, an early ipsilateral response mediated by the oligosynaptic pathway through the pons that is ipsilateral to the stimulation site; and R2, a late bilateral response mediated through the polysynaptic medullary pathway 
$(9,10)$. The $\mathrm{R} 1$ and $\mathrm{R} 2$ components of the recovery cycle can be evaluated by applying two stimuli to the supraorbital nerve at varying interstimulus intervals (ISI) to determine the excitability of brainstem interneurons (10).

In the present study, we aimed to evaluate and compare the $\mathrm{R} 1$ and $\mathrm{R} 2$ responses of blink reflex and $\mathrm{R} 2$ recovery cycle in patients with $\mathrm{BEB}$ and the healthy controls.

\section{Materials and Methods}

\section{Study Design}

In this prospective case-control study, patients with a diagnosis of blepharospasm $(n=18)$, according to the diagnostic criteria (i.e., the presence of stereotyped, bilateral, and synchronous orbicularis oculi spasms inducing narrowing/closure of the eyelids and the presence of effective sensory tricks or increased blinking) (4), were recruited from were recruited from the Movement Disorder Clinic of Rasoul-Akram Hospital in Tehran, Iran. Meanwhile, 18 individuals with no history of neurologic conditions, who were matched in terms of age and gender with the case group, were enrolled in the control group. This study was performed during $2017-$ 2018 in Rasoul-Akram Hospital, Tehran, Iran.

The current study was approved by the Research Committee of Iran University of Medical Sciences (ethical code: IR.IUMS.SMD.REC.1396.9411158003). All participants were informed about the study objectives and methods and signed the informed consent forms before participating in the study.

\section{Blink Reflex and R2 Recovery Cycle}

For patients who had received BTX, the blink reflex test was performed three months after injections. The electromyography (EMG) recordings were documented and calculated by Natus UltraPro S100 EMG system. To evaluate the blink reflex, the subjects were requested to relax on a bed in a room with moderate temperature, while slowly closing their eyes. Data were recorded by bipolar stimulation with a double channel recorder. The surface nerve conduction study (NCS) electrodes were placed bilaterally on the orbicularis oculi muscle. The device sensitivity was $200 \mathrm{mV}$, and the duration of each stimulus was $0.2 \mathrm{~ms}$. The $\mathrm{R} 1, \mathrm{R} 2$, and R2' responses were obtained by stimulating the supraorbital nerve on each side, and the amplitude and latency of each wave were calculated and recorded.

We evaluated the $\mathrm{R} 2$ recovery cycle for each subject. The R2 recovery cycle was measured by applying bilateral stimulation to the supraorbital nerves. Stimulation was applied at ISIs of 200, 300, 500, and $1000 \mathrm{~ms}$. To minimize habituation, we considered 50-60 seconds of rest between the stimuli. The intensity of all stimuli was recorded at the lowest intensity, reaching the response threshold. The R2 recovery index was calculated for each bilateral stimulation at each ISI, according to the data recorded by the device. This index measures the ratio of $\mathrm{R} 2$ amplitude in the first stimulation (condition) to the $\mathrm{R} 2$ amplitude in the second stimulation (test).

\section{Statistical Analysis}

Qualitative and descriptive analysis for demographic characteristics were reported as mean \pm standard deviation (SD) $(11,12)$. To compare variables between case and control groups, independent sample t-test was used $(13,14)$. The Kolmogorov-Smirnov test was used to assess the normal distribution of variables (14). Pearson's correlation test was performed to show correlation of variables and outcomes $(\mathbf{1 5}, \mathbf{1 6})$. The twotailed P-value $<0.05$ was considered as statistically significant. All statistical analysis was performed using Statistical Package for the Social Sciences, version 22.0 (SPSS Inc., Chicago, Ill., USA).

\section{Results}

In this study, 36 participants (18 females and 18 males) with an average age of 57.61 years (range: 39-72 years) were recruited. The mean age of the BEB group ( 9 females and 9 males) was $57.67 \pm 9.60$ years, and the mean age of the control group ( 9 females and 9 males) was $57.67 \pm 10.01$ years. There was no significant difference in terms of age or gender between the two groups. Also, the mean duration of disease was $3.67 \pm 3.21$ years in BEB group. Family history of the disease was negative in all participants. Only one patient had a positive drug history of neuroleptics (5\%). Overall, $77 \%$ of patients had been previously treated with BTX $(\mathrm{n}=14)$.

Table 1. Blink reflex responses

\begin{tabular}{|c|c|c|c|c|}
\hline \multicolumn{2}{|c|}{ Values } & BFS $(n=18)$ & Control (n=18) & P-value \\
\hline \multirow{5}{*}{ Distal latency } & RT R1(ms) & $12.09 \pm 1.37$ & $11.82 \pm 0.95$ & 0.50 \\
\hline & RT R2(ms) & $36.56 \pm 5.23$ & $34.44 \pm 3.35$ & 0.16 \\
\hline & RT R2'(ms) & $37.63 \pm 5.69$ & $37.03 \pm 4.13$ & 0.72 \\
\hline & LT R1(ms) & $11.97 \pm 1.28$ & $13.57 \pm 7.20$ & 0.36 \\
\hline & LT R2(ms) & $37.45 \pm 5.31$ & $35.25 \pm 2.79$ & 0.13 \\
\hline
\end{tabular}




\begin{tabular}{|c|c|c|c|c|}
\hline \multicolumn{2}{|c|}{ Values } & BFS $(n=18)$ & Control $(n=18)$ & P-value \\
\hline & LT R2'(ms) & $38.06 \pm 5.42$ & $37.76 \pm 3.22$ & 0.84 \\
\hline \multirow{2}{*}{ Amplitude } & $\mathrm{R} 1$ & $0.27 \pm 0.54$ & $0.28 \pm 0.50$ & 0.70 \\
\hline & R2 & $0.16 \pm 0.32$ & $0.18 \pm 0.33$ & 0.87 \\
\hline \multirow{4}{*}{$\begin{array}{l}\text { R2 recovery } \\
\text { index }\end{array}$} & $1000 \mathrm{~ms}$ & $90.17 \pm 13.19$ & $68.88 \pm 11.05$ & $0.00^{* *}$ \\
\hline & $500 \mathrm{~ms}$ & $86.17 \pm 12.44$ & $41.22 \pm 8.65$ & $0.00^{* *}$ \\
\hline & $300 \mathrm{~ms}$ & $80.36 \pm 13.01$ & $16.99 \pm 11.56$ & $0.00^{* *}$ \\
\hline & $200 \mathrm{~ms}$ & $75.70 \pm 12.20$ & $12.57 \pm 10.27$ & $0.00^{* *}$ \\
\hline \multicolumn{2}{|c|}{ Stimulus Intensity (mA) } & $12.96 \pm 1.54$ & $13.20 \pm 1.89$ & 0.68 \\
\hline
\end{tabular}

** P-value $<0.05$

The results of blink reflex analysis and comparisons between the two groups have been summarized in Table 1. There were no significant differences in R1, $\mathrm{R} 2$, and R2' distal latencies between the groups $(P>0.05) \quad$ (Table 1). There was no significant difference in the amplitude of $\mathrm{R} 1$ or $\mathrm{R} 2$ response $(P>0.05)$ (Table 1). The stimulus intensities were $13.20 \pm 1.89 \mathrm{~mA}$ and $12.96 \pm 1.54 \mathrm{~mA}$ in the control and
BEB groups, respectively, indicating no significant difference $(P>0.05)$. please change number 2 to 1 (Table 1) The R2 recovery index was improved in all patients at all four ISIs $(1000,500,300$, and $200 \mathrm{~ms})$ (Table 1). The most remarkable improvement was observed at 200 and $300 \mathrm{~ms}$ intervals (75.70 vs. 12.57 and 80.36 vs. 16.99, respectively) (Figure 1).

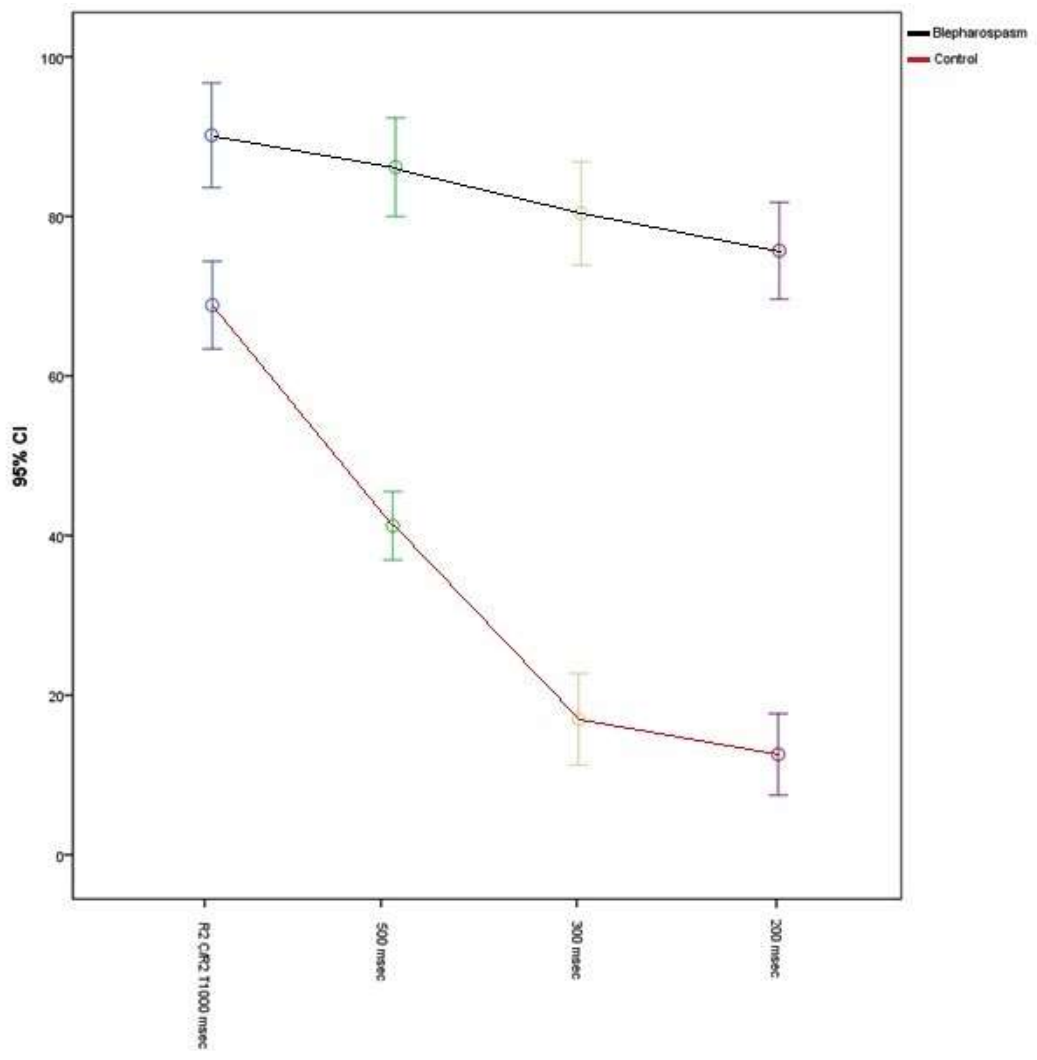

Figure 1. R2 Recovery Index

$\mathbf{R} 2$ recovery index in controls and patients with blepharospasm. Means are shown for the $\mathbf{R} 2$ amplitude at the first stimulus (condition) to the $\mathrm{R} 2$ amplitude at the second stimulation (test). Data is shown as Mean \pm Standard deviation (SD). X-axis: interstimulus intervals (ISI) in milliseconds. Y-axis: ratio of the conditioned to the tested amplitude of $R 2$ response in percent 


\section{Discussion}

In the current study, we evaluated the blink reflex at least three months after the injection of BTX for a more consistent investigation. However, the literature suggests that BTX injection has no significant effect on the excitability of brainstem interneurons, which mediate the $\mathrm{R} 2$ response of the blink reflex circuit $(2,6$, $7)$. In the present study, the stimulus intensity to elicit blink reflex responses was $12.96 \mathrm{~mA}$ in the BEB group and $13.2 \mathrm{~mA}$ in the control group, which is not significantly different. This intermediate stimulus intensity $(12 \mathrm{~mA})$ was also reported by Sommer et al. as the most appropriate intensity to indicate differences in responses between the control and BEB groups $(17,18)$.

The R1 and R2 components of blink reflex have been appraised in previous studies, and no significant difference has been reported between the BEB and control groups. Also, the peak amplitude of the cycles showed no significant difference between the BEB and control groups (10). In the present study, the R1 and R2 amplitudes were not significantly different between the BEB and control groups. Also, there was no significant difference between the BEB and control groups in the distal latencies of R1, R2, and R2' circuits, which is in line with previous studies $(5,18)$.

The significant increase in R2 recovery has been reported in parkinsonism, hemifacial spasm, cervical dystonia, and focal dystonia $(10,19)$, including BEB at all stimulation intervals $(10,18,19)$. In this regard, Schwingenschuh et al. demonstrated the significant enhancement of the R2 recovery component in BEB patients, compared to the controls and patients with atypical presentations of BEB, suggesting psychogenic blepharospasm (5). Our findings revealed that the R2 recovery reflex was enhanced in BEB patients. Previous studies have attributed the increased blink reflex excitability in blepharospasm to the abnormal function of brainstem interneurons. This finding suggests that a pathology in the basal ganglia dopamine system mediates the blink reflex excitability by inhibiting the superior colliculus $(7,20,21)$.

However, recent studies have shown that BEB is more likely to be associated with abnormal neural pathways $(22,23)$, and owing to its different manifestations, different pathologies have been suggested for the disease (22). The different abnormalities in the excitability of various $\mathrm{R} 2$ recovery cycles suggest two hypotheses. First, BEB has different subtypes with different severities and pathophysiological mechanisms; and second, different groups of BEB with different severities have a common pathophysiology (1). In this regard, Ferrazzano et al. suggested a common pathophysiological mechanism. However, the recovery cycle of the blink reflex deteriorated with the disease progression (24).

Previous studies on patients with blepharospasm have indicated an abnormal $\mathrm{R} 2$ recovery index, as well as hemi-facial spasm, post-facial syndrome, segmental cranio-cervical dystonia, and torticollis $(9,10,19)$. In this regard, Yaman et al. found the most significant difference in the $\mathrm{R} 2$ recovery index at an interval of $200 \mathrm{~ms}$ and the smallest difference at an interval of 800 ms (19). Similarly, our comparison of the blink reflex recovery cycle between the $\mathrm{BEB}$ and healthy groups at ISIs of 200, 300, 500, and $1000 \mathrm{~ms}$ indicated the significant improvement of the R2 recovery index in the BEB group, which was the most significant at intervals of 200 and $300 \mathrm{~ms}$. Some previous studies have reported similar results, indicating an abnormal $\mathrm{R} 2$ recovery index in the majority of blepharospasm patients $(5,9,10,18,24)$. The upper limit of normal for the index was defined as the mean value plus 2 or 2.5 SDs of the values obtained from healthy subjects in some studies. In a study by Schwingenschuh et al., $90 \%$ of patients with blepharospasm showed an abnormal R2 recovery index. They argued that the R2 recovery index is a helpful diagnostic tool to distinguish BEB from psychogenic blepharospasm, with $90 \%$ specificity and $100 \%$ sensitivity (5).

The mean duration of BEB was 3.67 \pm 3.21 years in the patients, which was not significantly correlated with the $\mathrm{R} 2$ recovery index. However, further investigations with a longer follow-up period are needed to compare the R2 recovery index over time. Also, we found no significant correlation between the age of disease onset and the R2 recovery index. This finding was supported by earlier reports, which indicated no significant correlation between the R2 index and the duration of disease, severity of disease, or age (10).

\section{Conclusion}

The findings of this study revealed that the R2 recovery reflex was higher in $\mathrm{BEB}$ patients than the controls. Therefore, it can be a suitable tool for diagnosing and differentiating BEB from its mimics.

\section{Acknowledgment}

The authors thank the patients and their families for making this study possible through their participation.

\section{Ethical considerations}

Ethical issues (Including plagiarism, informed consent, misconduct, data fabrication and/or falsification, double publication and/or submission, redundancy, etc.) have been completely observed by the authors.

\section{Funding and support}

This research resulted from an independent research without receiving any financial support.

\section{Conflict of Interest}


Authors declared no conflict of interest.

\section{References}

1. Defazio G, Hallett M, Jinnah HA, Conte A, Berardelli A. Blepharospasm 40 years later. J Move Disorder Soc. 2017;32(4):498-509. [DOI:10.1002/mds.26934] [PMID] [PMCID]

2. Karp BI, Alter K. Botulinum toxin treatment of blepharospasm, orofacial/oromandibular dystonia, and hemifacial spasm. Seminars Neurol. 2016;36(1):84-91. [DOI:10.1055/s-00361571952] [PMID]

3. Ramirez-Castaneda J, Jankovic J. Long-term efficacy, safety, and side effect profile of botulinum toxin in dystonia: a 20 -year follow-up. J Int Soc Toxinol. 2014;90:344-8. [DOI:10.1016/j.toxicon.2014.07.009] [PMID]

4. Defazio G, Hallett M, Jinnah HA, Berardelli A. Development and validation of a clinical guideline for diagnosing blepharospasm. Neurol. 2013;81(3):236-40. [DOI:10.1212/WNL.0b013e31829bfdf6] [PMID] [PMCID]

5. Schwingenschuh $\mathrm{P}$, Katschnig P, Edwards MJ, et al. The blink reflex recovery cycle differs between essential and presumed psychogenic blepharospasm. Neurol. 2011;76(7):610-4. [DOI:10.1212/WNL.0b013e31820c3074] [PMID] [PMCID]

6. Conte A, Fabbrini G, Belvisi D, Marsili L, Di Stasio F, Berardelli A. Electrical activation of the orbicularis oculi muscle does not increase the effectiveness of botulinum toxin type $\mathrm{A}$ in patients with blepharospasm. Europ J Neurol. 2010;17(3):449-55. 1331.2009.02840.x] [PMID]

7. Grandas F, Traba A, Alonso F, Esteban A. Blink reflex recovery cycle in patients with blepharospasm unilaterally treated with botulinum toxin. Clin Neuropharmacol. 1998;21(5):307-11.

8. Tolosa E, Montserrat L, Bayes A. Blink reflex studies in focal dystonias: enhanced excitability of brainstem interneurons in cranial dystonia and spasmodic torticollis. J Move Disorder Soc. 1988;3(1):61-9. [DOI:10.1002/mds.870030108] [PMID]

9. Aramideh M, Eekhof JL, Bour LJ, Koelman JH, Speelman JD, Ongerboer de Visser BW. Electromyography and recovery of the blink reflex in involuntary eyelid closure: a comparative study. J Neurol Neurosurg Psychiatr. 1995;58(6):692-8. [DOI:10.1136/jnnp.58.6.692] [PMID] [PMCID]

10. Eekhof JL, Aramideh M, Bour LJ, Hilgevoord AA, Speelman HD, Ongerboer de Visser BW. Blink reflex recovery curves in blepharospasm, torticollis spasmodica, and hemifacial spasm. Muscle Nerve. 1996;19(1):10-5 https://doi.org/10.1002/(SICI)1097-

4598(199601)19:1<10::AID-MUS2>3.0.CO;2-B

[DOI:10.1002/(SICI)10974598(199601)19:13.0.CO;2-B]

11. Mahdizadeh F, Mehraban AH, Faranoush M, Amini M, Mehdizadeh M. Fatigue in Children with Cancer: Reliability and Validity of the Persian Version of Child, Parent, and Staff Fatigue Scale. Asia-Pacific Journal of Oncology Nursing. 2020;7(2):174.

[DOI:10.4103/apjon.apjon_44_19] [PMCID]

[PMID]

12. Mehdizadeh M, Fereshtehnejad S-M, Goudarzi S, Moshtagh A, Dehghanian Nasrabadi F, Habibi SAH, et al. Validity and Reliability of Short-Form McGill Pain Questionnaire-2 (SF-MPQ-2) in Iranian People with Parkinson's Disease. Parkinson's Disease. 2020;2020. [DOI:10.1155/2020/2793945] [PMID] [PMCID]

13. Mehdizadeh M, Martinez-Martin P, Habibi SA, Nikbakht N, Alvandi F, Bazipoor P, et al. The association of balance, fear of falling, and daily activities with drug phases and severity of disease in patients with Parkinson. Basic and clinical neuroscience. 2019;10(4):355 [DOI:10.32598/bcn.9.10.295] [PMID] [PMCID]

14. Mehdizadeh M, Mehraban AH, Zahediyannasab R. The effect of group-based occupational therapy on performance and satisfaction of stroke survivors: pilot trail, neuro-occupational view. Basic and clinical neuroscience. 2017;8(1):69. [DOI:10.15412/J.BCN.03080109] [PMID] [PMCID]

15. Taghizadeh G, Martinez-Martin P, Meimandi M, Habibi SAH, Jamali S, Dehmiyani A, et al. Barthel Index and modified Rankin Scale: Psychometric properties during medication phases in idiopathic Parkinson disease. Annals of Physical and Rehabilitation 2019. [DOI:10.1016/j.rehab.2019.08.006] [PMID]

16. Azad A, Mohammadinezhad T, Taghizadeh G, Lajevardi L. Clinical assessment of activities of daily Hewite Gite athis Attigle: Validation of the Persian version of Katz Index. Medical journal of the Islamic Republic of Iran. 2017; 31:30. [DOI:10.18869/mjiri.31.30] [PMID] [PMCID]

17. Sommer M, Ferbert A. The stimulus intensity modifies the blink reflex recovery cycle in healthy subjects and in blepharospasm. Int Federation Clin Neurophysiol. 2001;112(12):2293-9. [DOI:10.1016/S1388-2457(01)00701-5]

18. Yaman M, Sahin S, Kiziltan ME. Blink reflex recovery in central and peripherally originated movement disorders of the cranio-cervical area: a 
comparative study. Electromyograph Clin Neurophysiol. 2009;49(1):19-25.

19. Basso MA, Powers AS, Evinger C. An explanation for reflex blink hyperexcitability in Parkinson's disease. I. Superior Colliculus. J Neurosci. 1996;16(22):7308-17.

[DOI:10.1523/JNEUROSCI.16-22-07308.1996] [PMID] [PMCID]

20. Curra A, Bagnato S, Berardelli A. Recent findings in cranial and cervical dystonia: how they help us to understand the pathophysiology of dystonia. Supp Clin Neurophysiol. 2006;58:257-65. [DOI:10.1016/S1567-424X(09)70074-1]

21. Hwang CJ, Eftekhari K. Benign essential blepharospasm: what we know and what we don't. Int Ophthalmol Clin. 2018;58(1):11-24. [DOI:10.1097/IIO.0000000000000207] [PMID]
22. Quartarone A, Sant'Angelo A, Battaglia F, et al. Enhanced long-term potentiation-like plasticity of the trigeminal blink reflex circuit in blepharospasm. J Neurosci Soc Neurosci. 2006;26(2):716-21. [DOI:10.1523/JNEUROSCI.3948-05.2006] [PMID] [PMCID]

23. Defazio G, Conte A, Gigante AF, et al. Clinical heterogeneity in patients with idiopathic blepharospasm: A cluster analysis. Parkinsonism Relat Disorder. 2017;40:64-8. [DOI:10.1016/j.parkreldis.2017.04.014] [PMID]

24. Ferrazzano G, Conte A, Gigante A, Defazio G, Berardelli A, Fabbrini G. Disease progression in blepharospasm: a 5-year longitudinal study. Europ J Neurol. 2019;26(2):268-73. [DOI:10.1111/ene.13832] [PMID] 